Farbod Fahimi*

\title{
Vision-Based CubeSat Closed-Loop Formation Control in Close Proximities
}

https://doi.org/10.1515/nleng-2017-0147

Received August 8, 2017; accepted February 21, 2018.

\begin{abstract}
A vision based formation and attitude controller has been derived and simulated for the formation keeping of two 3U CubeSats. Four markers are installed on the leader CubeSat. Two cameras are installed on the follower CubeSat. An efficient vision based pose estimation method is used to estimate the pose of the follower with respect to the leader. A Higher-Order Sliding Mode (HOSM) exact differentiator with finite-time convergence is derived to estimate the rate of the follower's pose parameters. The follower's pose and its time rate are fedback to HOSM formation and attitude controllers to correct any gradual drift in formation and pose of the follower. The simulations show the effectiveness of the approach, and its feasibility on existing hardware.
\end{abstract}

\section{Introduction}

Small satellites are lighter and less expensive to launch, and if one satellite fails it can be replaced with less cost. Yet, they can be used in constellations to perform important missions. For example, several projects at NASA can strongly benefit from the utilization of multiple satellites in orbit: Global Precipitation Measurement Constellation, Leonardo-BRDF, GPS Occultation Constellation, Magnetospheric Constellation, Global Ionosphere-ThermosphereMesosphere Constellation, etc. That is why many research institutions, including NASA's Goddard Space Flight Center have been studying and designing and testing tools that enable the use of constellations of small satellites [1].

The architecture of the formation of small satellites have been studied by some researchers [2, 3]. A three-body problem modeling and an up to date model of the Dydimos binary asteroid has been used to highlighting benefits and limitations of stable and unstable formation fly-

\footnotetext{
*Corresponding Author: Farbod Fahimi, Associate Professor, Mechanical and Aerospace Engineering Department, University of Alabama in Huntsville, Huntsville, Alabama 35801, U.S.A.

E-mail: farbod.fahimi@uah.edu
}

ing orbits [4]. To design a realistic formation architecture, a multitude of operational scenarios such as desaturation, detumble, thruster firing, and target acquisition/recovery have been considered [5].

Another aspect of formation control of small satellites is the prescription and planning of the needed formation maneuvers at different situations. Planning of $n$-impulse fuel-optimal maneuvers for establishment and reconfiguration of spacecraft formations are considered for circular and elliptic orbits [6]. Collision avoidance maneuvers for a swarm of satellites in formation, which return to their original configuration after a threat has passed, is considered in [7] using biological rules of avoidance.

Other researchers focused on the control of separation distances and orientation of satellites in formations. The interaction between magnets and superconductors has been used as a mechanism to affect the relative pose of small satellites in a formation [8]. This mechanism, known as magnetic flux pinning, has been used with a Proportional-Integral-Derivative control law for choosing an optimal configuration for the spacecraft formation during a rest-to-rest reorientation maneuver [9]. Precision dual spacecraft formation flying has been proposed to enable a "virtual" telescope by utilizing the Constrained Model Predictive Control approach [10]. It was shown by simulations that the formation control using aerodynamic drag forces are feasible at altitudes between $500 \mathrm{~km}$ and $700 \mathrm{~km}$ [11]. The station-keeping, reconfiguration and collision avoidance of spacecraft in formation around eccentric reference orbits have been addressed by development of an LQR/APF control scheme [12]. The software that enables rendezvous, proximity operations, and docking for CubeSat formation flying has been designed in [13] using a dual-inertial state Extended Kalman Filter, and a fueloptimal state-space impulsive guidance and control algorithm.

In this research, to address the GN\&C needs of close formation flying of small satellites, a vision based pose estimation combined with robust Higher-Order Sliding Mode (HOSM) controllers is explored. First, a vision based pose estimation method is briefly introduced $[14,15]$. Then, a HOSM exact differentiator with finite-time convergence is explained. Next, a HOSM formation controller and a HOSM attitude controller are presented. Finally, the vision system 


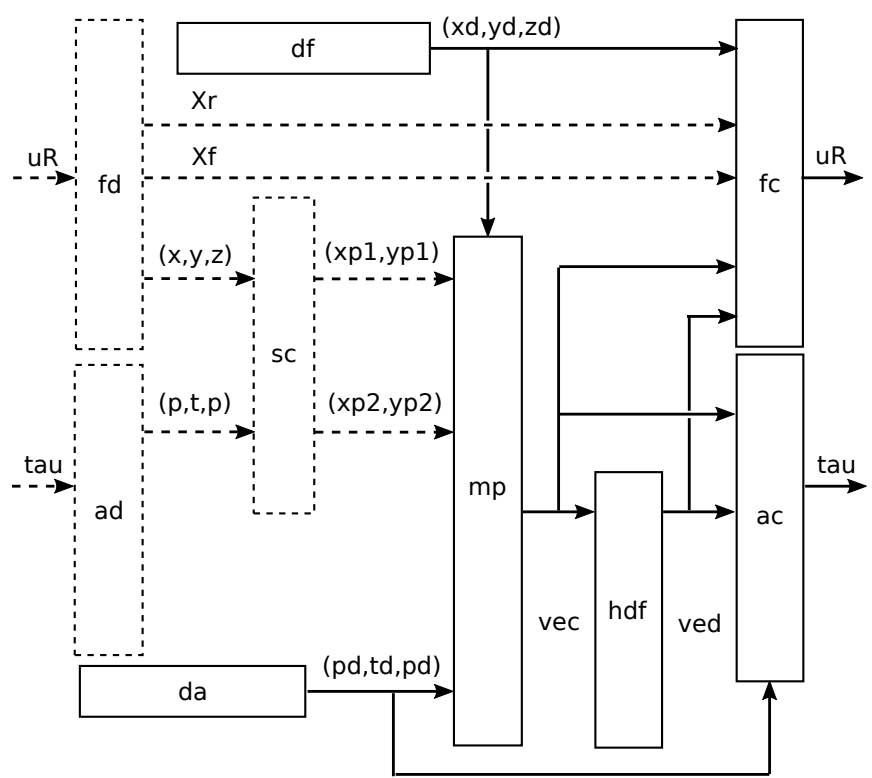

Fig. 1: The vision based formation control architecture

is integrated with the controllers and the performance of the whole system is shown via simulations.

\section{System Architecture}

The building blocks of the vision based control system and their relations are shown in Fig. 1. The blocks that are shown in solid lines are parts of the controller. The blocks shown in dashed lines represent the mathematical models of the actual hardware that are used for simulations. For the real implementation, these blocks will be replaced by the actual CubeSats and their sensors.

A user defines the "desired formation" $\left(x^{d}, y^{d}, z^{d}\right)$ and "desired attitude" $\left(\phi^{d}, \theta^{d}, \psi^{d}\right)$ of the follower with respect to the leader according to the application at hand. Four recognizable markers $j=1, \ldots, 4$ are installed on the leader. Two cameras $c_{1}$ and $c_{2}$, installed on the follower, capture the images of the markers on the leader. The image pixel coordinates of the markers $\left[\left(x_{p j}, y_{p j}\right)_{c_{1}}\right.$ and $\left.\left(x_{p j}, y_{p j}\right)_{c_{2}}\right]$ are derived by processing the camera images. These image pixel coordinates and the desired pose of the follower are fed to the "Mirage vision based pose estimation" algorithm, which provides the actual pose of the follower $(x, y, z, \phi, \theta, \psi)$. The actual pose is differentiated by the "HOSM differentiator" in real time. The actual pose, its rate, and the desired pose are fed to the closedloop "HOSM formation controller" and "HOSM attitude controller," which determine the thruster forces $\left(\mathbf{u}_{f}\right)$ and reaction wheel torques $(\boldsymbol{\tau})$ to correct any drift in formation and attitude of the follower. In the following, each building block is presented and discussed.

\section{Desired Formation and Desired Attitude}

The leader and follower CubeSats are shown in Fig. 2. There are two poses shown for the follower. One (on the left) is the desired position of the follower CubeSat with respect to the leader CubeSat. The desired pose of the follower is represented by a spatial frame $\left\{f^{d}\right\}$, while the current pose of the leader is denoted by the frame $\{r\}$. Based on the application at hand, a user defines his/her desired relative formation $\left(x^{d}, y^{d}, z^{d}\right)$ and orientation $\left(\phi^{d}, \theta^{d}, \psi^{d}\right)$ of the follower CubeSat with respect to the leader. Knowing the desired pose of the follower frame $\left\{f^{d}\right\}$ with respect to the leader frame $\{r\}$, one can define the known transformation matrix between the two frames $\mathbf{T}_{r}^{f^{d}}$.

$$
\mathbf{T}_{f^{d}}^{r}=\left[\begin{array}{ccc|c}
\mathbf{R}_{z}\left(\psi^{d}\right) \mathbf{R}_{y}\left(\theta^{d}\right) \mathbf{R}_{x}\left(\phi^{d}\right) & x^{d} \\
y^{d} \\
\hline \begin{array}{llll}
z^{d} \\
\hline
\end{array} & 0 & 1
\end{array}\right], \quad \mathbf{T}_{r}^{f^{d}}=\left(\mathbf{T}_{f^{d}}^{r}\right)^{-1}
$$

Here, $\mathbf{R}_{z}\left(\right.$.), $\mathbf{R}_{y}\left(\right.$.), and $\mathbf{R}_{x}($.) are the $3 \times 3$ rotation matrices about the $z$, $y$, and $x$ axes, respectively.

\section{Mirage Vision Based Pose Estimation}

\subsection{Pose estimation methodology}

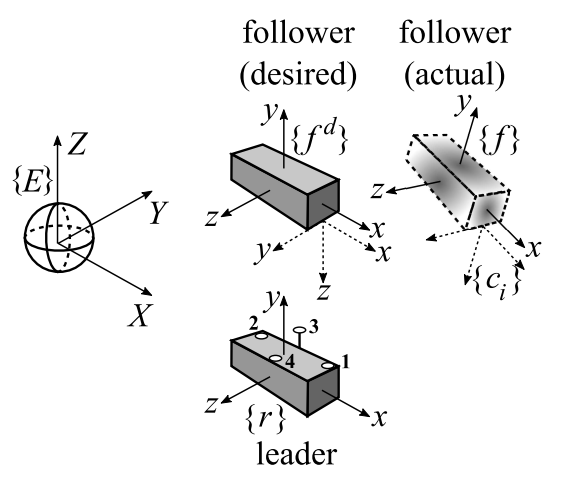

Fig. 2: The leader and follower CubeSats with body frames 
Figure 2 shows the leader and follower CubeSats. Two poses are shown for the follower. The one on the left is the desired (ideal) position of the follower CubeSat with respect to the leader CubeSat. However, the follower has drifted away from its desired pose with respect to the leader. The actual pose of the follower after the drift (shown on the right) is denoted by a spatial frame $\{f\}$.

Note that the transformation matrix $\mathbf{T}_{r}^{f^{d}}$ is known from Eq. (1). If one can determine the transformation between the frames $\{f\}$ and $\left\{f^{d}\right\}$ (i.e., the matrix $\mathbf{T}_{f^{d}}^{f}$ ), then, the actual relative pose of the follower $\{f\}$ with respect to the leader $\{r\}$ can be determined by the following simple transformation.

$$
\mathbf{T}_{f}^{r}=\left(\mathbf{T}_{f^{d}}^{f} \mathbf{T}_{r}^{f^{d}}\right)^{-1}
$$

The Mirage pose estimation method directly calculates the elements of matrix $\mathbf{T}_{f^{d}}^{f}$ via an analytical linear noniterative solution, when two (or more) cameras are used.

The calculation of $\mathbf{T}_{f^{d}}^{f}$ involves the comparison of two images: the desired image and the actual image, which are defined in the following. Four non-planar markers (with known geometry) are installed on the leader (points 1 through 4 in Fig. 2). Two cameras are installed on the follower, but only one of them, represented by frame $\left\{c_{i}\right\}$, is shown in Fig. 2 . The camera $\left\{c_{i}\right\}(i=1,2)$ generates images of the markers. The image seen by the camera when the follower is at its desired pose is called the desired image. On the other hand, the image that the camera sees when the follower is in its actual pose is called the actual image.

The difference in the pixel coordinates of marker $j$ $(j=1, \ldots, 4)$ in the actual and desired images of camera $c_{i}(i=1,2)$ is a function of $\mathbf{T}_{f d}^{f}$. This difference in pixel coordinates is denoted by the following equation.

$$
\mathbf{e}_{j}^{c_{i}}=\left[\begin{array}{l}
x_{p_{j}}-x_{p_{j}}^{d} \\
y_{p_{j}}-y_{p_{j}}^{d}
\end{array}\right]_{c_{i}}
$$

where $\left(x_{p_{j}}, y_{p_{j}}\right)_{c_{i}}$ and $\left(x_{p_{j}}^{d}, y_{p_{j}}^{d}\right)_{c_{i}}$ are the pixel coordinates of marker $j$ in camera $i$ in the actual and desired image, respectively. Note that both the actual and the desired pixel coordinates are known. The actual pixel coordinates are found by extracting the pixel coordinates of the markers in the actual image via image processing. The desired pixel coordinates are found as follows.

After markers are physically installed on the leader, the Euclidean position vector of marker $j$ with respect to the origin of $\{r\}$ is denoted by $\left(\mathbf{r}_{r}\right)_{j}=\left[r_{x}, r_{y}, r_{z}, 1\right]_{j}^{T}$. The desired 3D projection of marker $j$ in the camera space $\left(\mathbf{p}_{c_{i}}^{d}\right)_{j}=$ $\left[\begin{array}{lll}p_{c_{i} X}^{d} & p_{c_{i} y}^{d} & p_{c_{i} z}^{d}\end{array}\right]_{j}^{T}$ is found by a series of transformation applied to the vector $\mathbf{r}_{r}$.

$$
\left(\mathbf{p}_{j}^{d}\right)_{c_{i}}=\mathbf{K}_{c_{i}} \mathbf{T}_{f^{d}}^{c_{i}} \mathbf{T}_{r}^{f^{d}}\left(\mathbf{r}_{r}\right)_{j}
$$

Here, the $4 \times 4$ matrix $\mathbf{T}_{f^{d}}^{c_{i}}$ transforms any vector from frame $\left\{f^{d}\right\}$ to frame $\left\{c_{i}\right\}$, and the $3 \times 4$ matrix $\mathbf{K}_{c_{i}}$ contains the intrinsic camera parameters (focal length, principle points and distortion parameters), which are available a priori via camera calibration. Then, the desired pixel coordinates are obtained.

$$
\left[\begin{array}{c}
x_{p_{j}}^{d} \\
y_{p_{j}}^{d}
\end{array}\right]_{c_{i}}=\left[\begin{array}{l}
p_{j x}^{d} / p_{j z}^{d} \\
p_{j y}^{d} / p_{j z}^{d}
\end{array}\right]_{c_{i}}
$$

Therefore, the pixel error $\mathbf{e}_{j}^{c_{i}}$ is known. If a relation between the pixel error $\mathbf{e}_{j}^{c_{i}}$ and the transformation matrix $\mathbf{T}_{f^{d}}^{f}$ is established, that relation can be used to solve for the elements of $\mathbf{T}_{f d}^{f}$. The derivation of such a relation is explained in the following.

First, the pixel error $\mathbf{e}_{j}^{c_{i}}$ can be calculated based on the coordinates in the 3D camera space.

$$
\mathbf{e}_{j}^{c_{i}}=\left[\begin{array}{l}
x_{p_{j}}-x_{p_{j}}^{d} \\
y_{p_{j}}-y_{p_{j}}^{d}
\end{array}\right]_{c_{i}}=\left[\begin{array}{l}
\frac{p_{j x}}{p_{j z}}-\frac{p_{j x}^{d}}{p_{j z}^{d}} \\
\frac{p_{j y}}{p_{j z}}-\frac{p_{j y}^{d}}{p_{j z}^{d}}
\end{array}\right]_{c_{i}}
$$

The actual 3D projection of marker $j$ in the camera space $\left\{c_{i}\right\}$ is denoted.

$$
\left(\mathbf{p}_{j}\right)_{c_{i}}=\mathbf{K}_{c_{i}} \mathbf{T}_{f}^{c_{i}} \mathbf{T}_{r}^{f}\left(\mathbf{r}_{r}\right)_{j}
$$

Equation (7) is rewritten by incorporating the fact that $\mathbf{T}_{r}^{f}=$ $\mathbf{T}_{f^{d}}^{f^{d}} \mathbf{T}_{r}^{f^{d}}$.

$$
\left(\mathbf{p}_{j}\right)_{c_{i}}=\mathbf{K}_{c_{i}} \mathbf{T}_{f}^{c_{i}} \mathbf{T}_{f^{d}}^{f} \mathbf{T}_{r}^{f^{d}}\left(\mathbf{r}_{r}\right)_{j}
$$

Note that there are no unknowns in Eq. (4) and the only unknowns in Eq. (8) are the twelve elements of the matrix $\mathbf{T}_{f^{d}}^{f}$.

$$
\mathbf{T}_{f^{d}}^{f}=\left[\begin{array}{cccc}
t_{1} & t_{4} & t_{7} & t_{10} \\
t_{2} & t_{5} & t_{8} & t_{11} \\
t_{3} & t_{6} & t_{9} & t_{12} \\
0 & 0 & 0 & 1
\end{array}\right]
$$

Equation (9) is substituted in Eq. (8). The result of the substitution and Eq. (4) are expanded to obtain the components of $\left(\mathbf{p}_{j}\right)_{c_{i}}$ and $\left(\mathbf{p}_{j}^{d}\right)_{c_{i}}$. The resulting components are substituted in Eq. (6). After very involved manipulations, which can be found in [15], the following relation is found between the pixel error $\mathbf{e}_{j}^{c_{i}}$ and the elements of $\mathbf{T}_{f^{d}}^{f}$.

$$
\mathbf{e}_{j}^{c_{i}}=\mathbf{V}_{j}^{c_{i}} \mathbf{t}_{f^{d}}^{f}+\mathbf{Y}_{j}^{c_{i}}
$$


where $\mathbf{V}^{c_{i}}$ is a $2 \times 12$ matrix and $\mathbf{Y}^{c_{i}}$ is a $2 \times 1$ vector with known values. For the detailed formulation of these parameters, see [15]. The $12 \times 1$ vector $\mathbf{t}_{f^{d}}^{f}$ contains all 12 unknown elements of the transformation matrix $\mathbf{T}_{f^{d}}^{f}$.

$$
\mathbf{t}_{f^{d}}^{f}=\left[t_{1}, t_{2}, t_{3}, t_{4}, t_{5}, t_{6}, t_{7}, t_{8}, t_{9}, t_{10}, t_{11}, t_{12}\right]^{T}
$$

Note that Eq. (10) includes 2 equations and 12 unknowns and cannot be solved in its current form. However, this equation is valid for camera $c_{i}(i=1,2)$ and marker $j$ $(j=1, \ldots, 4)$. For this case, Eq. (10) is written 8 times, covering the combinations of 2 cameras $c_{1}$ and $c_{2}$ and 4 markers.

$$
\left[\begin{array}{c}
\mathbf{e}_{1}^{c_{1}} \\
\mathbf{e}_{2}^{c_{1}} \\
\mathbf{e}_{3}^{c_{1}} \\
\mathbf{e}_{4}^{c_{1}} \\
\mathbf{e}_{1}^{c_{2}} \\
\mathbf{e}_{2}^{c_{2}} \\
\mathbf{e}_{3}^{c_{2}} \\
\mathbf{e}_{4}^{c_{2}}
\end{array}\right]=\left[\begin{array}{c}
\mathbf{V}_{1}^{c_{1}} \\
\mathbf{V}_{2}^{c_{1}} \\
\mathbf{V}_{3}^{c_{1}} \\
\mathbf{V}_{4}^{c_{1}} \\
\mathbf{V}_{1}^{c_{2}} \\
\mathbf{V}_{2}^{c_{2}} \\
\mathbf{V}_{3}^{c_{2}} \\
\mathbf{V}_{4}^{c_{2}}
\end{array}\right] \mathbf{t}_{f^{d}}^{f}+\left[\begin{array}{c}
\mathbf{Y}_{1}^{c_{1}} \\
\mathbf{Y}_{2}^{c_{1}} \\
\mathbf{Y}_{3}^{c_{1}} \\
\mathbf{Y}_{4}^{c_{1}} \\
\mathbf{Y}_{1}^{c_{2}} \\
\mathbf{Y}_{2}^{c_{2}} \\
\mathbf{Y}_{3}^{c_{2}} \\
\mathbf{Y}_{4}^{c_{2}}
\end{array}\right], \quad \text { or } \quad \mathbf{e}=\mathbf{V t}_{f^{d}}^{f}+\mathbf{Y}
$$

Equation (12) forms a linear system of 16 equations in terms of 12 unknown elements of $\mathbf{t}_{f d}^{f}$, which can be easily solved in the Least-Square sense.

$$
\mathbf{t}_{f^{d}}^{f}=\left(\mathbf{V}^{T} \mathbf{V}\right)^{-1}\left(\mathbf{V}^{T}(\mathbf{e}-\mathbf{Y})\right)
$$

Now that the vector $\mathbf{t}_{f^{d}}^{f}$ is calculated, the transformation matrix $\mathbf{T}_{f^{d}}^{f}$ is found. Then, $\mathbf{T}_{f}^{r}$ is calculated using Eq. (2). The elements of $\mathbf{T}_{f}^{r}$ are related to the pose parameters of the follower $\left(\mathbf{q}=[x, y, z]^{T}\right.$ and $\left.\boldsymbol{\Phi}=[\phi, \theta, \psi]^{T}\right)$.

$$
\mathbf{T}_{f}^{r}=\left[\begin{array}{cll|c}
\mathbf{R}_{z}(\psi) \mathbf{R}_{y}(\theta) \mathbf{R}_{x}(\phi) & y \\
& & z \\
\hline 0 & 0 & 0 & 1
\end{array}\right]
$$

Therefore, knowing $\mathbf{T}_{f}^{r}$ one can solve for $\mathbf{q}=[x, y, z]^{T}$ and $\boldsymbol{\Phi}=[\phi, \theta, \psi]^{T}$.

\subsection{The simulated cameras}

The block "simulated cameras" calculates the "actual pixel coordinates" of the marker $j(j=1, \ldots, 4)$ for simulation only using the simulated pose of the follower CubeSat mathematical model. The actual 3D projection of marker $j$ in the camera space is found using Eq. (7) and $\mathbf{T}_{r}^{f}=\left(\mathbf{T}_{f}^{r}\right)^{-1}$. Finally, the simulated pixel coordinates are obtained as follows.

$$
\left[\begin{array}{c}
x_{p_{j}} \\
y_{p_{j}}
\end{array}\right]_{c_{i}}=\left[\begin{array}{l}
p_{j x} / p_{j z} \\
p_{j y} / p_{j z}
\end{array}\right]_{c_{i}}
$$

\section{HOSM Differentiator}

The pose vector $\mathbf{Q}=[\mathbf{q}, \boldsymbol{\Phi}]^{T}$ is not sufficient for feedback control. The rate of the pose vector $\dot{\mathbf{Q}}$ is also required. The conventional differentiators provide asymptotic convergence. When the rate of a vector changes rapidly with time, the asymptotic convergence causes a phase lag, which is not good for robust control.

Here a Higher-Order Sliding Model (HOSM) differentiator is introduced that provides finite-time (as opposed to asymptotic) convergence to the exact derivative (in the absence of measurement noise). The HOSM differentiator is obtained as follows.

The time variable measured signal $\mathbf{Q}(t)$ is considered. It is desired to calculate $\dot{\mathbf{Q}}(t)$ in real-time. A real-time estimate of the measured signal is defined as $\tilde{\mathbf{Q}}(t)$. At any given time the estimation error is defined.

$$
\mathbf{y}_{Q}=\tilde{\mathbf{Q}}(t)-\mathbf{Q}(t)
$$

The derivative of the estimation error is given as follows.

$$
\dot{\mathbf{y}}_{Q}=\dot{\tilde{\mathbf{Q}}}-\dot{\mathbf{Q}}
$$

The rate of the estimated measurement $\dot{\tilde{\mathbf{Q}}}$ is considered as a control input that affects the dynamics of the estimation error $\mathbf{y}_{Q}$.

$$
\mathbf{v}=\dot{\tilde{\mathbf{Q}}}
$$

Therefore, the dynamics of the estimation error is obtained.

$$
\dot{\mathbf{y}}_{Q}=-\dot{\mathbf{Q}}+\mathbf{v}
$$

A control law for $\mathbf{v}$ is desired such that it can drive both $\mathbf{y}_{Q}$ and $\dot{\mathbf{y}}_{Q}$ to zero in finite-time. If both $\mathbf{y}_{Q}$ and $\dot{\mathbf{y}}_{Q}$ are driven to zero in finite-time, then, $\tilde{\mathbf{Q}} \rightarrow \mathbf{Q}$ and $\dot{\mathbf{Q}} \rightarrow \dot{\mathbf{Q}}$, at which point the exact derivative of $\mathbf{Q}$ is obtained. The following control law achieves the goal.

$$
\begin{gathered}
\mathbf{v}=\lambda_{1} \operatorname{sign}(\tilde{\mathbf{Q}}-\mathbf{Q})^{T}|\tilde{\mathbf{Q}}-\mathbf{Q}|^{\frac{1}{2}}+\mathbf{q}_{1} \\
\dot{\mathbf{q}}_{1}=-\lambda_{2} \operatorname{sign}(\tilde{\mathbf{Q}}-\mathbf{Q})
\end{gathered}
$$

Here, $\lambda_{1}$ and $\lambda_{2}$ are positive constants.

The differentiator is used as follows. The initial value of the measured signal $\mathbf{Q}(0)$ is known. The initial value of the estimated measured signal is set to $\tilde{\mathbf{Q}}(0)=\mathbf{Q}(0)$. The control signals $\mathbf{q}_{1}$ and $\mathbf{v}$ are found by integrating Eq. (21) and calculating Eq. (20), respectively. The control signal $\mathbf{v}$ is applied to the dynamic system in Eq. (18), which is integrated to obtain $\tilde{\mathbf{Q}}$. The obtained $\mathbf{\mathbf { Q }}$ is fedback for control in Eqs. (20) and (21), and the process continues in time. In the meantime, the control signal $\mathbf{v}$ is the estimated derivative of the measured signal. 


\section{Formation Dynamic Model}

\subsection{Governing equations for simulations}

The coordinate axes shown in Fig. 2 are considered. Let $\mathbf{x}_{r}^{r}$ and $\mathbf{x}_{f}^{r}$ denote the position of the leader and the follower satellites with respect to the geocentric system $(X, Y, Z)$, but expressed along the moving axes $(x, y, z)$ of frame $\{r\}$ at the location of the leader satellite. Note that axes $x, y$, and $z$ are radial, along track, and cross-track directions, respectively, defined by Hill and used in [2].

It is known that the effect of the follower's thrusters force $\mathbf{u}_{f}$ on the acceleration of the follower $\ddot{\mathbf{x}}_{f}^{r}$ is described by the follower's equation of the motion.

$$
m_{f} \ddot{\mathbf{x}}_{f}^{r}=\mathbf{F}_{E f}^{r}+\mathbf{F}_{r f}^{r}+\mathbf{R}_{f}^{r} \mathbf{u}_{f}+\mathbf{F}_{d f}^{r}
$$

In this equation, $m_{f}$ is the mass of the follower satellite; $\mathbf{F}_{E f}^{r}$ is the pulling force from the Earth on the follower satellite; $\mathbf{F}_{r f}^{r}$ is the pulling force from the leader satellite on the follower satellite; and $\mathbf{F}_{d f}^{r}$ is the resultant perturbing forces on the follower satellite. All vectors, except $\mathbf{u}_{f}$, have a superscript $r$, which indicates that they are written along the axes of the moving coordinate $(x, y, z)$ of the frame $\{r\}$. The thrust vector $\mathbf{u}_{f}$ is expressed in frame $\{f\}$, and $\mathbf{R}_{f}^{r}$ transforms a vector from frame $\{f\}$ to frame $\{r\}$.

This equation is integrated for simulations in the "formation dynamic model" block to provide $\mathbf{x}_{f}^{r}$. The trajectory of the leader $\mathbf{x}_{r}^{r}$ is directly defined by the user.

\subsection{Governing equations for control law derivation}

Here, to achieve a precise formation, the relative position of the follower with respect to the leader will be controlled. Therefore, the dynamic equations of motion must be written as a relation between $\ddot{\mathbf{q}}$ and the control forces from the follower's thrusters $\mathbf{u}_{f}$.

The acceleration of the follower satellite can be written in terms of the acceleration of the leader satellite.

$$
\ddot{\mathbf{x}}_{f}^{r}=\ddot{\mathbf{x}}_{r}^{r}+\dot{\boldsymbol{\omega}}_{r}^{r} \times \mathbf{q}+\boldsymbol{\omega}_{r}^{r} \times\left(\boldsymbol{\omega}_{r}^{r} \times \mathbf{q}\right)+2 \boldsymbol{\omega}_{r}^{r} \times \dot{\mathbf{q}}+\ddot{\mathbf{q}}
$$

where $\mathbf{q}$ is the relative distance of the follower with respect to the leader along the moving axes $(x, y, z)$ of frame $\{r\}$, and $\boldsymbol{\omega}_{r}^{r}$ is the instantaneous rate of rotation of the leader around the Earth expressed along the axes of $\{r\}$. For the derivation of the controller, the "nominal" situation is used, in which the resultant perturbing forces on the follower satellite are neglected $\left(\mathbf{F}_{d f}^{r}=\mathbf{0}\right)$ and the nominal mass of the satellite is used $\left(\hat{m}_{f}\right)$.
Equation (22) is substituted in Eq. (23), and the result is solved for $\ddot{\mathbf{q}}$. The following is obtained

$$
\ddot{\mathbf{q}}=\hat{\mathbf{f}}_{x}+\hat{b}_{x} \mathbf{u}_{f}
$$

where

$$
\begin{aligned}
\hat{\mathbf{f}}_{x} & =-\left(\ddot{\mathbf{x}}_{r}^{r}+\dot{\boldsymbol{\omega}}_{r}^{r} \times \mathbf{q}+\boldsymbol{\omega}_{r}^{r} \times\left(\boldsymbol{\omega}_{r}^{r} \times \mathbf{q}\right)+2 \boldsymbol{\omega}_{r}^{r} \times \dot{\mathbf{q}}\right) \\
& +\left(\hat{\mathbf{F}}_{E f}^{r}+\hat{\mathbf{F}}_{r f}^{r}\right) / \hat{m}_{f} \\
\hat{b}_{x} & =\left(1 / \hat{m}_{f}\right) \mathbf{R}_{f}^{r}
\end{aligned}
$$

in which $\hat{\mathbf{F}}_{E f}^{r}$ and $\hat{\mathbf{F}}_{r f}^{r}$ are calculated using $\hat{m}_{f}$.

Equation (24) describes the direct effect of the follower's thrust $\mathbf{u}_{f}$ on the relative motion of the follower with respect to the leader (q). This equation will be used for derivation of the formation controller.

\subsection{Implementation notes}

A controller that is derived based on Eq. (24) needs the measurement of the realtime acceleration of the leader $\left(\ddot{\mathbf{x}}_{r}^{r}\right)$, the relative position of the follower with respect to the leader $(\mathbf{q})$, and its rate $(\dot{\mathbf{q}})$. The acceleration $\ddot{\mathbf{x}}_{r}^{r}$ can be measured by an accelerometer on board of the leader satellite, and can be broadcast to the follower satellite. The relative position $\mathbf{q}$ is measured by the vision system, and its rate $\dot{\mathbf{q}}$ is observed by the HOSM differentiator.

\section{Attitude Dynamic Model}

\subsection{Governing equations of motion}

The ability to correct the satellite's attitude is important, because after long periods of time, the external perturbing forces and moments will eventually rotate the satellite bodies. This may cause the loss of sight for visionbased formation correction maneuvers. Therefore, an attitude controller must be used to realign the satellites precisely when necessary.

A CubeSat body axes $\left(x_{B}, y_{B}, z_{B}\right)$ and its three orthogonal reaction wheels are shown in Fig. 3. The moment components $\boldsymbol{\tau}=\left[\tau_{1 x}, \tau_{2 y}, \tau_{3 z}\right]^{T}$ are exerted by the motors of

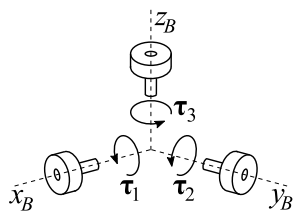

Fig. 3: A CubeSat with three orthogonal reaction wheels 
the reaction wheels on the CubeSat's body. The following equations describes the rotational dynamics of the CubeSat body.

$$
\begin{aligned}
\left(\mathbf{I}_{B}+\sum_{i=1}^{3} \mathbf{B}_{i} \mathbf{I}_{i}\right) \dot{\boldsymbol{\omega}}_{B} & =\boldsymbol{\tau}-\boldsymbol{\omega}_{B} \times \mathbf{I}_{B} \boldsymbol{\omega}_{B} \\
& -\sum_{i=1}^{3} \mathbf{B}_{i}\left(\boldsymbol{\omega}_{B} \times \mathbf{I}_{i} \boldsymbol{\omega}_{i}\right)+\boldsymbol{\tau}_{D} \\
\dot{\boldsymbol{\Phi}} & =\mathbf{E} \boldsymbol{\omega}_{B}
\end{aligned}
$$

The rotational dynamics of the reaction wheel are described by the following equation, in which $\theta_{i}(i=1,2,3)$ is the angular position of reaction wheel $i$.

$$
\left[\begin{array}{c}
I_{1 x x} \ddot{\theta}_{1} \\
I_{2 y y} \ddot{\theta}_{2} \\
I_{3 z z} \ddot{\theta}_{3}
\end{array}\right]=-\boldsymbol{\tau}-\sum_{i=1}^{3} \mathbf{A}_{i}\left(\mathbf{I}_{i} \dot{\boldsymbol{\omega}}_{B}+\boldsymbol{\omega}_{B} \times \mathbf{I}_{i} \boldsymbol{\omega}_{i}\right)
$$

The derivation and the definition of the notation used in the above equations are found in the Appendix.

In summary, Eqs. (27), (28), and (29) are the complete dynamic model of the CubeSat and reaction wheels as a system, which are inserted in the "attitude dynamic model” block in Fig. 1. At any simulation step, the reaction wheel driving moments are known. One can calculate the CubeSat's angular acceleration vector $\dot{\boldsymbol{\omega}}_{B}$ using Eq. (27). Also, the reaction wheel driving moments and the CubeSat's angular acceleration vector $\dot{\boldsymbol{\omega}}_{B}$ just obtained from Eq. (27) can be used with Eq. (29) to calculate the reaction wheel's angular acceleration $\left(\ddot{\theta}_{1}, \ddot{\theta}_{2}, \ddot{\theta}_{3}\right)$. Integrating these angular accelerations will provide $\boldsymbol{\omega}_{B}$ and $\left(\dot{\theta}_{1}, \dot{\theta}_{2}, \dot{\theta}_{3}\right)$ for the next time step. The integration can go on by repeating the above steps.

\subsection{Governing equations for control law derivations}

Equation (27) is written in a more concise form.

$$
\dot{\boldsymbol{\omega}}_{B}=\hat{\mathbf{f}}_{\omega}+\hat{\mathbf{b}}_{\omega} \boldsymbol{\tau}
$$

where

$$
\begin{aligned}
\hat{\mathbf{b}}_{\omega} & =\left(\hat{\mathbf{I}}_{B}+\sum_{i=1}^{3} \mathbf{B}_{i} \hat{\mathbf{I}}_{i}\right)^{-1} \\
\hat{\mathbf{f}}_{\omega} & =-\hat{\mathbf{b}}_{\omega}\left(\boldsymbol{\omega}_{B} \times \hat{\mathbf{I}}_{B} \boldsymbol{\omega}_{B}+\sum_{i=1}^{3} \mathbf{B}_{i}\left(\boldsymbol{\omega}_{B} \times \hat{\mathbf{I}}_{i} \boldsymbol{\omega}_{i}\right)\right)
\end{aligned}
$$

are calculated using the "nominal" values of the system parameters. The following outputs are defined for the control system in Eq. (30).

$$
\mathbf{y}=\boldsymbol{\Phi}-\boldsymbol{\Phi}^{d}
$$

where $\boldsymbol{\Phi}^{d}$ is the desired orientation of the satellite, which in general can be a function of time. A controller must be derived that can stabilize the output $\mathbf{y}$ of the system in Eq. (30) and its time derivative at zero vector in finite time. The dynamics of the output $\mathbf{y}$ is derived for control design in the following.

The second time derivative of the surface variable is calculated.

$$
\begin{aligned}
\ddot{\mathbf{y}} & =\ddot{\boldsymbol{\Phi}}-\ddot{\boldsymbol{\Phi}}^{d} \\
& =\mathbf{E} \dot{\boldsymbol{\omega}}_{B}+\dot{\mathbf{E}} \boldsymbol{\omega}_{B}-\ddot{\boldsymbol{\Phi}}^{d} \\
& =\mathbf{E}\left(\hat{\mathbf{f}}_{\omega}+\hat{\mathbf{b}}_{\omega} \boldsymbol{\tau}\right)+\dot{\mathbf{E}} \boldsymbol{\omega}_{B}-\ddot{\boldsymbol{\Phi}}^{d} \\
& =\left(\mathbf{E} \hat{\mathbf{f}}_{\omega}+\dot{\mathbf{E}} \boldsymbol{\omega}_{B}-\ddot{\boldsymbol{\Phi}}^{d}\right)+\left(\mathbf{E} \hat{\mathbf{b}}_{\omega}\right) \boldsymbol{\tau}
\end{aligned}
$$

If the notations $\hat{\mathbf{f}}_{\phi}=\mathbf{E} \hat{\mathbf{f}}_{\omega}+\dot{\mathbf{E}} \boldsymbol{\omega}_{B}-\ddot{\boldsymbol{\Phi}}^{d}$ and $\hat{\mathbf{b}}_{\phi}=\mathbf{E} \hat{\mathbf{b}}_{\omega}$ are adopted, the model for attitude control derivation becomes.

$$
\ddot{\mathbf{y}}=\hat{\mathbf{f}}_{\phi}+\hat{\mathbf{b}}_{\phi} \boldsymbol{\tau}
$$

The above equation is used for the derivation of a HOSM attitude controller.

\section{HOSM Formation and Attitude Controllers}

In this section a HOSM controller is derived for both the formation controller and the attitude controller. Note that the concise form of the dynamics of the two systems in Eq. (24) and Eq. (34) fit the following standard form.

$$
\ddot{\boldsymbol{\sigma}}=\hat{\mathbf{f}}+\hat{\mathbf{b}} \mathbf{u}
$$

Here, a HOSM controller is derived based on the standard model in Eq. (35), which is interchangeable between systems in Eqs. (24) and (34) by using the correct $\boldsymbol{\sigma}, \hat{\mathbf{f}}, \hat{\mathbf{b}}$, and u.

\subsection{Nominal control}

A change of state variables such as $\boldsymbol{\sigma}=\mathbf{z}_{1}$ and $\dot{\boldsymbol{\sigma}}=\mathbf{z}_{2}$ and change of the control signal such as $\hat{\mathbf{w}}=\hat{\mathbf{f}}+\hat{\mathbf{b}} \hat{\mathbf{u}}$ are applied to the output system in Eq. (35). The following chain form is obtained.

$$
\begin{aligned}
& \dot{\mathbf{z}}_{1}=\mathbf{z}_{2} \\
& \dot{\mathbf{z}}_{2}=\hat{\mathbf{w}}
\end{aligned}
$$

It can be shown that the following feedback control law can stabilize the system in Eq. (36) at zero vector in finite 
time [16],

$$
\hat{w}_{j}=\sum_{i=1}^{2} k_{i j} \operatorname{sign}\left(z_{i j}\right)\left|z_{i j}\right|^{v_{i j}}, \quad j=1,2,3
$$

where $v_{1 j}=3 / 5$ and $v_{2 j}=3 / 4$, and the control gains $k_{i j}$ are found such that the following characteristic polynomial $\left(p^{2}+k_{2 j} p+k_{1 j}=0, j=1,2,3\right)$ is Hurwitz. The above formulation is used as explained in the following. At each time step, the measurement of the real-time errors and their rates provide the feedback signals $\mathbf{z}_{1}$ and $\mathbf{z}_{2}$.

$$
\begin{aligned}
& \mathbf{z}_{1}=\boldsymbol{\sigma} \\
& \mathbf{z}_{2}=\dot{\boldsymbol{\sigma}}
\end{aligned}
$$

Then, the nominal control is calculated using Eq. (37). Finally the equivalent control is calculated by inverting the relation $\hat{\mathbf{w}}=\hat{\mathbf{f}}+\hat{\mathbf{b}} \mathbf{u}$.

$$
\hat{\mathbf{u}}=\hat{\mathbf{b}}^{-1}(-\hat{\mathbf{f}}+\hat{\mathbf{w}})
$$

The above "nominal" control works accurately if the system model has no uncertainty, that is if $\hat{\mathbf{f}}$ and $\hat{\mathbf{b}}$ are exact. However, normally, the real system is represented by $\mathbf{f}$ and $\mathbf{b}$, which are different due to modeling and parameters uncertainties. In the following, the nominal control is amended to account for such uncertainties.

\subsection{Extension to uncertain systems}

The relation between the terms of the nominal system model and those of the actual system are defined as $\mathbf{f}=\hat{\mathbf{f}}+\tilde{\mathbf{f}}$ and $\mathbf{b}=\hat{\mathbf{b}}+\tilde{\mathbf{b}}$, in which $\tilde{\mathbf{f}}$ and $\tilde{\mathbf{b}}$ are bounded. The following equation represents the real system.

$$
\ddot{\boldsymbol{\sigma}}=(\hat{\mathbf{f}}+\tilde{\mathbf{f}})+(\hat{\mathbf{b}}+\tilde{\mathbf{b}}) \hat{\mathbf{u}}
$$

Noting that $\mathbf{z}_{2}=\dot{\boldsymbol{\sigma}}$ and substituting Eq. (40) in the above relation, one obtains.

$$
\dot{\mathbf{z}}_{2}=\tilde{\mathbf{f}}-\tilde{\mathbf{b}} \hat{\mathbf{b}}^{-1} \hat{\mathbf{f}}+\left(\mathbf{I}+\tilde{\mathbf{b}} \hat{\mathbf{b}}^{-1}\right) \hat{\mathbf{w}}
$$

To make sure that $\dot{\mathbf{z}}_{2} \rightarrow 0$ even in the presence of uncertainty, the nominal control $\hat{\mathbf{w}}$ in Eq. (42) is replaced by

$$
\begin{aligned}
\mathbf{w} & =\hat{\mathbf{w}}-G \operatorname{sign}(\mathbf{s}) \\
\dot{\mathbf{Z}}_{\mathrm{aux}} & =-\hat{\mathbf{w}}
\end{aligned}
$$

where $\mathbf{s}=\mathbf{z}_{2}+\mathbf{z}_{\mathrm{aux}}$, and $G$ is a positive constant. The gain $G$ must be determined such that it is large enough to compensate for uncertain terms. This gain is determined using a Lyponuv design method.

\subsection{Lyaponuv gain design}

A Lyaponuv function is defined as $V=\frac{1}{2} \mathbf{s}^{T} \mathbf{s}$. The gain $G$ must be determined such that $\dot{V}=\mathbf{s}^{T} \dot{\mathbf{s}} \leq 0$ for all $\mathbf{s}$. Because $\dot{\mathbf{s}}=\dot{\mathbf{z}}_{2}+\dot{\mathbf{z}}_{\text {aux }}=\dot{\mathbf{z}}_{2}-\hat{\mathbf{w}}, \dot{V}$ is derived as follows.

$$
\begin{aligned}
\dot{V} & =\mathbf{s}^{T}\left[\tilde{\mathbf{f}}-\tilde{\mathbf{b}} \hat{\mathbf{b}}^{-1} \hat{\mathbf{f}}\right]+\mathbf{s}^{T}\left(\tilde{\mathbf{b}} \hat{\mathbf{b}}^{-1}\right) \hat{\mathbf{w}} \\
& -\mathbf{s}^{T} G \operatorname{sign}(\mathbf{s})+\mathbf{s}^{T}\left(-\tilde{\mathbf{b}} \hat{\mathbf{b}}^{-1}\right) G \operatorname{sign}(\mathbf{s})
\end{aligned}
$$

To design the controller gain $G$, the following bounds are defined for the uncertain terms.

$$
\begin{gathered}
\left\|\tilde{\mathbf{f}}-\tilde{\mathbf{b}} \hat{\mathbf{b}}^{-1} \hat{\mathbf{f}}\right\| \leq \rho \\
\left\|-\tilde{\mathbf{b}} \hat{\mathbf{b}}^{-1}\right\|=\left\|\tilde{\mathbf{b}} \hat{\mathbf{b}}^{-1}\right\| \leq 1-\alpha
\end{gathered}
$$

Note that $\alpha \approx 1$. Knowing that $\mathbf{c}^{T} \mathbf{d} \leq\|\mathbf{c}\|\|\mathbf{d}\|$ and $\|\mathbf{s}\| \leq$ $\mathbf{s}^{T} \operatorname{sign}(\mathbf{s})$, and using Eq. (45), one can obtain the following.

$$
\begin{aligned}
\dot{V} & \leq\|\mathbf{s}\| \rho+\|\mathbf{s}\|(1-\alpha)\|\hat{\mathbf{w}}\|-G\|\mathbf{s}\|+G(1-\alpha)\|\mathbf{s}\| \\
& \leq-(\alpha G-\rho-(1-\alpha)\|\hat{\mathbf{w}}\|)\|\mathbf{s}\|
\end{aligned}
$$

Equation (47) implies that, if the control gain $G$ is selected to meet the following requirement,

$$
G \geq \frac{\rho+(1-\alpha)\|\hat{\mathbf{w}}\|+\eta}{\alpha}
$$

where $\eta>0$, then, $\dot{V} \leq-\eta\|\mathbf{s}\|$, or $\dot{V}$ is negative for all $\mathbf{s}$ and $V=\frac{1}{2} \mathbf{s}^{T} \mathbf{s}$ vanishes to zero despite bounded uncertainties in the system defined by Eq. (46), provided that the following control law is used.

$$
\mathbf{u}=\hat{\mathbf{b}}^{-1}(-\hat{\mathbf{f}}+\hat{\mathbf{w}}-G \operatorname{sign}(\mathbf{s}))
$$

Based on the formation dynamic model in Eq. (24), the above control law is used as the "HOSM formation controller" by setting $\hat{\mathbf{f}}=\hat{\mathbf{f}}_{x}, \hat{\mathbf{b}}=\hat{\mathbf{b}}_{x}, \mathbf{z}_{1}=\mathbf{q}, \mathbf{z}_{2}=\dot{\mathbf{q}}$, and $\mathbf{u}=\mathbf{u}_{f}$. Also, based on the attitude dynamic model in Eq. (34), the above control law is used as the "HOSM attitude controller" by setting $\hat{\mathbf{f}}=\hat{\mathbf{f}}_{\phi}, \hat{\mathbf{b}}=\hat{\mathbf{b}}_{\phi}, \mathbf{z}_{1}=\mathbf{y}, \mathbf{z}_{2}=\dot{\mathbf{y}}$, and $\mathbf{u}=\boldsymbol{\tau}$. The term $\hat{\mathbf{w}}$ is always calculated using the generic Eq. (37).

\section{Simulation}

A CubeSat similar to the one designed in [17] is considered. The moment of inertia of the satellite is $\hat{\mathbf{I}}_{B} \approx \operatorname{diag}(0.0067,0.0333,0.0333) \mathrm{kg} \cdot \mathrm{m}^{2}$. The reactions wheels are SI's 3-wheel orthogonal model [18], whose estimated mass moment of inertia are $\hat{\mathbf{I}}_{1}=\operatorname{diag}(2.392,1.247,1.247) \times 10^{-5} \mathrm{~kg} \cdot \mathrm{m}^{2}$, $\hat{\mathbf{I}}_{2}=\operatorname{diag}(1.247,2.392,1.247) \times 10^{-5} \mathrm{~kg} \cdot \mathrm{m}^{2}$, and $\hat{\mathbf{I}}_{3}=\operatorname{diag}(1.247,1.247,2.392) \times 10^{-5} \mathrm{~kg} \cdot \mathrm{m}^{2}$. 

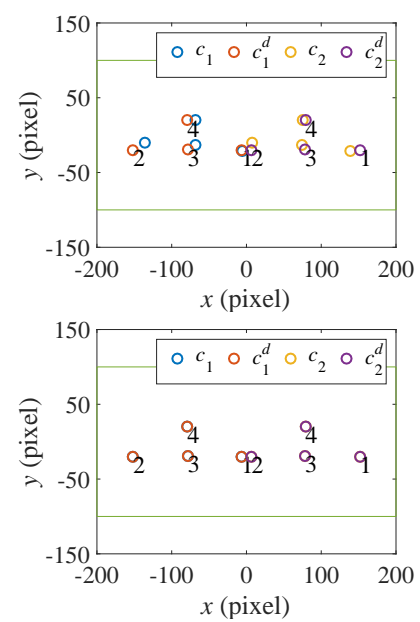

Fig. 4: Left: The images of the markers in the cameras at the beginning of the simulation. Right: The images of the markers in the cameras at the end of the simulation.

The effect of the resultant perturbing moment $\boldsymbol{\tau}_{D}$ is neglected during the very short duration of the controller's motion correction. An amount of $5 \%$ uncertainty has been assumed for the moments of inertia for the worst case. The total nominal mass of the follower is $\hat{m}_{f}=5 \mathrm{~kg}$, while the end of life mass after usage of all propellant of $m_{f}=4.64$ $\mathrm{kg}$ is used for simulation.

An in-track formation is assumed for the simulations. The desired roll, pitch, yaw of the follower with respect to the leader are all zeros $\left(\phi^{d}=\theta^{d}=\psi^{d}=0^{\circ}\right)$. However, a desired separation of $y^{d}=-5 \mathrm{~m}$ along the $y$ direction of the follower is assumed, while $x^{d}=z^{d}=0 \mathrm{~m}$.

When the formation/attitude correction is turned on, the follower has an orientation defined by $\phi(0)=5^{\circ}$, $\theta(0)=5^{\circ}$, and $\psi(0)=-5^{\circ}$. Also, $x(0)=0 \mathrm{~m}, y(0)=5.5 \mathrm{~m}$, and $z(0)=0 \mathrm{~m}$.

The images of the leader's markers in the follower's cameras at the beginning of the simulation, when the follower is not at the desired formation, are shown in the left plot of Fig. 4. The actual image, calculated by the simulated cameras, is different from the desired image, calculated by the internals of the Mirage vision based pose estimation algorithm. This is because at the start of the simulation, the follower is not at the desired formation location.

The images of the leader's markers in the follower's cameras at the end of the simulation, when the follower reaches its desired formation, are shown in the right plot of Fig. 4. It can be seen that the actual image, calculated by the simulated cameras, coincides with the desired image, calculated by the internals of the Mirage vision based pose estimation algorithm. This is because at the end of the
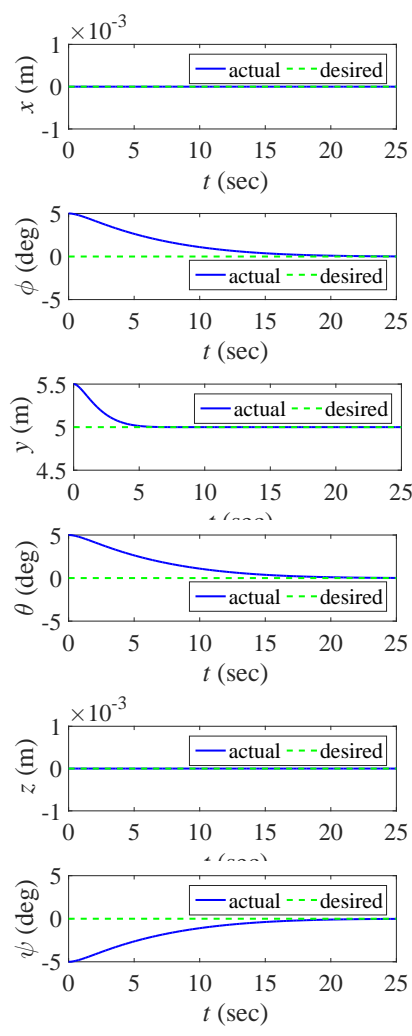

Fig. 5: Relative pose components

simulation, the follower is exactly at the desired formation location.

The relative position components of the follower with respect to the leader are shown in the left column of Fig. 5. The $x$ and $z$ components start and remain at their desired value of zero meters. The $y$ component, which needed correction, goes from $5.5 \mathrm{~m}$ to the desired value of $5 \mathrm{~m}$. The relative attitude components of the follower with respect to the leader are shown in the right column of Fig. 5. All components start with a $5^{\circ}$ error in pose. They converge to their desired values of zero degrees.

The thrust components of the follower are shown in the left column of Fig. 6. The in-plane formation for this simulation encounters 0.5 meters of drift each month [2]. The total impulse for the $0.5 \mathrm{~m}$ correction is $p=\int_{0}^{t_{f}} u_{2} d t=$ 0.17 N.s, where $t_{f}$ is the time at which the formation error is less than $0.001 \mathrm{~m}$. Assume that the CubeSat is using the hydrazine propulsion module presented in [19], which has a total life of 760 N.s of impulse. The thrusters can afford $4470=(760 / 0.17)$ corrections, which, at the rate of one correction per month, lasts 372 years. The maximum needed thrust is less than $2.8 \mathrm{~N}$, which is less than the maximum provided by the assumed hydrazine propulsion module [19]. The right column of Fig. 6 shows the torques of the follower's reaction wheels. All moments are below 


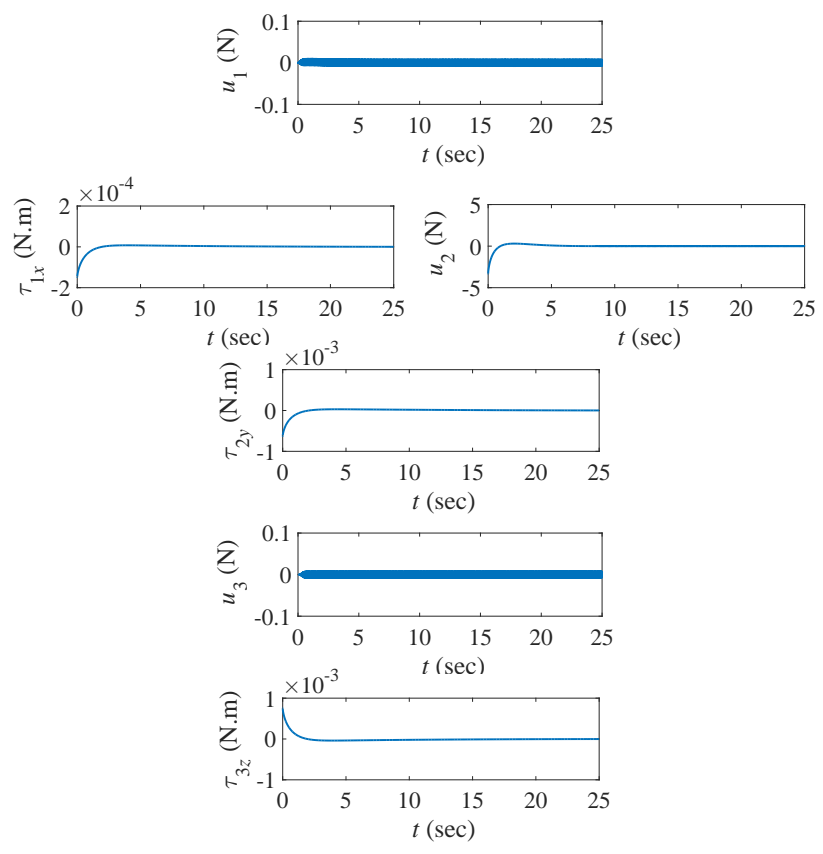

Fig. 6: Follower's thrust and reaction wheel torques

the maximum capability of the SI's orthogonal reaction wheels, which is $0.001 \mathrm{~N} . \mathrm{m}$ [18].

\section{Conclusions}

A vision based HOSM formation and attitude controller was derived and simulated for the formation keeping of two 3U CubeSats. Vision-based pose estimation in junction with a HOSM differentiator provide accurate states in absence of GPS signals. The distance of the leader and follower satellites are directly controlled for precise formation keeping. For precise attitude control, the attitude controller was derived based on the a multibody dynamic model that included the dynamics of the reaction wheels. The simulations show the effectiveness of the approaches, and their feasibility in light of the practical maximum thrust and reaction wheel torques, and the length of satellite mission.

Acknowledgement: This research was performed at NASA-MSFC under the Faculty Fellowship Program in Summer 2016, for which I thank Dr. Frank Six, the program director, and Dr. John Rakoczy, my supervisor, for their strong support.

\section{Appendix A: Derivations of the rotational dynamic model}

A CubeSat body axes $\left(x_{B}, y_{B}, z_{B}\right)$ and its three orthogonal reaction wheels are shown in Fig. 3. The moment vectors $\boldsymbol{\tau}_{1}, \boldsymbol{\tau}_{2}$, and $\boldsymbol{\tau}_{3}$ are exerted by the reaction wheels on the CubeSat's body. Note that these moment vectors include components that are the driving moments along the axes of the reaction wheels, as well as components that are internal (structural) moments perpendicular to the axes of the reaction wheels (Table1).

Table 1: Components of wheel moments

\begin{tabular}{c|c|c}
\hline $\boldsymbol{\tau}_{1}$ & \multicolumn{1}{|c}{$\boldsymbol{\tau}_{2}$} & $\boldsymbol{\tau}_{3}$ \\
\hline$\tau_{1 x}$ driving & $\tau_{2 x}$ internal & $\tau_{3 x}$ internal \\
$\tau_{1 y}$ internal & $\tau_{2 y}$ driving & $\tau_{3 y}$ internal \\
$\tau_{1 z}$ internal & $\tau_{2 z}$ internal & $\tau_{3 z}$ driving \\
\hline
\end{tabular}

The rotational equation of motion of the CubeSat body (excluding the reaction wheels) is written as follows.

$$
\mathbf{I}_{B} \dot{\boldsymbol{\omega}}_{B}+\boldsymbol{\omega}_{B} \times \mathbf{I}_{B} \boldsymbol{\omega}_{B}=\boldsymbol{\tau}_{1}+\boldsymbol{\tau}_{2}+\boldsymbol{\tau}_{3}-\boldsymbol{\tau}_{D}
$$

where $\mathbf{I}_{B}$ is the centroidal mass moment of inertia of the CubeSat, excluding the reaction wheels, and $\boldsymbol{\omega}_{B}$ is the angular velocity of the CubeSat body. Both $\mathbf{I}_{B}$ and $\boldsymbol{\omega}_{B}$ are expressed along the axes of the CubeSat's body frame $\left(x_{B}, y_{B}, z_{B}\right)$, which implies that the vector moments $\boldsymbol{\tau}_{1}, \boldsymbol{\tau}_{2}$, and $\boldsymbol{\tau}_{3}$ are also expressed in the body frame. $\boldsymbol{\tau}_{D}$ is the unknown (but bounded) resultant of all the external perturbing moments.

The equation of motion of the reaction wheel $i$ is

$$
\mathbf{I}_{i} \dot{\boldsymbol{\omega}}_{i}+\boldsymbol{\omega}_{B} \times \mathbf{I}_{i} \boldsymbol{\omega}_{i}=-\boldsymbol{\tau}_{i} \quad(i=1,2,3)
$$

where $\boldsymbol{\omega}_{1}=\boldsymbol{\omega}_{B}+\left[\dot{\theta}_{1}, 0,0\right]^{T}, \boldsymbol{\omega}_{2}=\boldsymbol{\omega}_{B}+\left[0, \dot{\theta}_{2}, 0\right]^{T}$, and $\boldsymbol{\omega}_{3}=\boldsymbol{\omega}_{B}+\left[0,0, \dot{\theta}_{3}\right]^{T}$. Also, $\theta_{i}$ is the angle of rotation of the reaction wheel $i$ about its shaft, and $\mathbf{I}_{i}$ is the diagonal mass moment of inertia tensor of the $i^{\text {th }}$ reaction wheel.

The twelve Eqs. (50) and (51) represent the dynamic state of the whole system, which include the six internal moments as listed in Table 1. The internal moments might be useful for the structural (or bearing) design of the reaction wheels, however, are not of interest for modeling the system for control design purposes. These six internal components of moment must be eliminated from twelve Eqs. (50) and (51). After the elimination, six $(12-6=6)$ equations remain, which represent the dynamics of the vectors $\boldsymbol{\omega}_{B}$ and $\left[\dot{\theta}_{1}, \dot{\theta}_{2}, \dot{\theta}_{3}\right]^{T}$. The elimination procedure follows. 
The following auxiliary matrices are defined to eliminate the internal moments in Eqs. (51).

$$
\mathbf{A}_{1}=\left[\begin{array}{lll}
1 & 0 & 0 \\
0 & 0 & 0 \\
0 & 0 & 0
\end{array}\right], \mathbf{A}_{2}=\left[\begin{array}{lll}
0 & 0 & 0 \\
0 & 1 & 0 \\
0 & 0 & 0
\end{array}\right], \mathbf{A}_{3}=\left[\begin{array}{lll}
0 & 0 & 0 \\
0 & 0 & 0 \\
0 & 0 & 1
\end{array}\right]
$$

Equation (29) is derived by simplifying the following summation: $\sum_{i=1}^{3} \mathbf{A}_{i}\left(\mathbf{I}_{i} \dot{\boldsymbol{\omega}}_{i}+\boldsymbol{\omega}_{B} \times \mathbf{I}_{i} \boldsymbol{\omega}_{i}\right)=-\sum_{i=1}^{3} \mathbf{A}_{i} \boldsymbol{\tau}_{i}$, where $\boldsymbol{\tau}=\left[\tau_{1 x}, \tau_{2 y}, \tau_{3 z}\right]^{T}$, and $\mathbf{I}_{i}$ is diagonal.

The following auxiliary matrices are defined to eliminate the internal moments in Eq. (50).

$$
\mathbf{B}_{1}=\left[\begin{array}{lll}
0 & 0 & 0 \\
0 & 1 & 0 \\
0 & 0 & 1
\end{array}\right], \mathbf{B}_{2}=\left[\begin{array}{lll}
1 & 0 & 0 \\
0 & 0 & 0 \\
0 & 0 & 1
\end{array}\right], \mathbf{B}_{3}=\left[\begin{array}{lll}
1 & 0 & 0 \\
0 & 1 & 0 \\
0 & 0 & 0
\end{array}\right]
$$

Note that $\boldsymbol{\tau}_{1}+\boldsymbol{\tau}_{2}+\boldsymbol{\tau}_{3}=\boldsymbol{\tau}+\sum_{i=1}^{3} \mathbf{B}_{i} \boldsymbol{\tau}_{i}$, which can be shown by expanding components of $\boldsymbol{\tau}_{i}$ (as shown in Table 1) on either side. Substituting for $\boldsymbol{\tau}_{1}+\boldsymbol{\tau}_{2}+\boldsymbol{\tau}_{3}$ from Eq. (50) and substituting for $\boldsymbol{\tau}_{i}$ from Eq. (51) and rearranging results in Eq. (27). Note that $\mathbf{I}_{i}$ is diagonal.

\section{References}

[1] Esper, J., Neeck, S., Slavin, J. A., Leitner, J., Wiscombe, W., and Bauer, F. H., 2003. "Nano/micro satellite constellations for Earth and space science". Acta Astronautica, 52(9-12), pp. 785-791.

[2] Sabol, C., Burns, R., and McLaughlin, C. A., 2001. "Satellite formation flying design and evolution". Journal of Spacecraft and Rockets, 38(2), pp. 270-278.

[3] Carpenter, J. R., Leitner, J., Folta, D., and Burns, R., 2003. "Benchmark problems for spacecraft formation flying missions". In AIAA Guidance, Navigation, and Control Conference and Exhibit. (AIAA 2003-5364).

[4] Capannolo, A., Lavagna, M., Ferrari, F., and Lunghi, P., 2016. "Nanosatellite formation flying to enhance science in binary asteroid environment". In Proceedings of the International Astronautical Congress, IAC. (IAC-16,B4,8,9,×35142).

[5] Schulte, P. Z., and Spencer, D. A., 2016. "Development of an integrated spacecraft guidance, navigation, control subsystem for automated proximity operations". Acta Astronautica, 118, pp. 168-186.

[6] Roscoe, C. W., Westphal, J. J., Griesbach, J. D., and Schaub, H., 2015. "Formation establishment and reconfiguration using differential elements in 12-perturbed orbits". Journal of Guidance, Control, and Dynamics, 38(9), pp. 1725-1740.

[7] Nag, S., and Summerer, L., 2013. "Behaviour based, autonomous and distributed scatter manoeuvres for satellite swarms". Acta Astronautica, 82(1), pp. 95-109.

[8] Wilson, W. R., Shoer, J. P., and Peck, M. A., 2009. "Demonstration of a magnetic locking flux-pinned revolute joint for use on cubesat-standard spacecraft”. In AIAA Guidance, Navigation, and Control Conference and Exhibit. (AIAA 2009-5904).
[9] Sorgenfrei, M. C., Jones, L. L., Joshi, S. S., and Peck, M. A., 2013. "Testbed validation of location-scheduled control of a reconfigurable flux-pinned spacecraft formation". Journal of Spacecraft and Rockets, 50(6), pp. 1235-1247.

[10] Park, S.-Y., Calhoun, P. C., Shah, N., and Williams, T. W., 2014. "Orbit design and control of technology validation mission for refractive space telescope in formation flying". In AIAA Guidance, Navigation, and Control Conference. (AIAA 20140451).

[11] Omar, S. R., and Wersinger, J., 2015. "Satellite formation control using differential drag". In 53rd AIAA Aerospace Sciences Meeting. (AIAA 2015-0002).

[12] Palacios, L., Ceriotti, M., and Radice, G., 2013. “Autonomous distributed LQR/APF control algorithms for CubeSat swarms manoeuvring in eccentric orbits". In Proceedings of the International Astronautical Congress, IAC, Vol. 7, pp. 5115-5121. (IAC-14-C1.5.7).

[13] Roscoe, C. W., Westphal, J. J., Lutz, S., and Bennett, T., 2015. "Guidance, navigation, and control algorithms for cubesat formation flying". In Advances in the Astronautical Sciences, Vol. 154, pp. 685-699. (AAS 15-113).

[14] Dinc, S., Fahimi, F., and Aygun, R., 2016. "Vision-based trajectory tracking for mobile robots using Mirage pose estimation method". IET Computer Vision, 10(5), August, pp. 450-458.

[15] Dinc, S., Fahimi, F., and Aygun, R., 2017. "Mirage: an $o(n)$ time analytical solution to 3D camera pose estimation with multi-camera support”. Robotica, pp.119. in press; Published online: 16 February 2017; DOI: https://doi.org/10.1017/S0263574716000874.

[16] Defoort, M., Floquet, T., Kokosy, A., and Perruquetti, W., 2009. "A novel higher order sliding mode control scheme". Systems and Control Letters, 58(2), pp. 102-108.

[17] Zeledon, R., and Peck, M., 2013. "Attitude dynamics and control of a 3 U CubeSat with electrolysis propulsion". In AIAA Guidance, Navigation, and Control (GNC) Conference, AIAA. (AIAA 2013-4943).

[18] Kjellberg, H. C., 2011. "Design of a CubeSat guidance, navigation, and control module”. Thesis for Master of Science in Engineering, The University of Texas at Austin, August. DOI: 10.13140/2.1.1469.7767.

[19] Schmuland, D. T., Masse, R. K., and Sota, C. G., 2011. "Hydrazine propulsion module for CubeSats". In Proceedings of the 25th Annual AIAA/USU Conference on Small Satellites, AIAA. (SSC11-X-4). 